\title{
THE NORMAL EXTENSIONS OF SUBGROUP TOPOLOGIES
}

\author{
BRADD CLARK AND VICTOR SCHNEIDER
}

\begin{abstract}
Let $H$ be a topological group contained in a group $G$. A topology which makes $G$ a topological group inducing the given topology on $H$ is called an extending topology. The set of all extending topologies forms a complete semilattice in the lattice of group topologies on $G$. The structure of this semilattice is studied by considering normal subgroups which intersect $H$ in the identity.
\end{abstract}

Let $G$ be a group and $\mathscr{L}$ the collection of continuous topologies on $G$. If $\left\{T_{\alpha}\right\}_{\alpha \in \Gamma}$ is any collection of topologies in $\mathscr{L}$, we can create a new topological group $\mathscr{G}=\Pi_{\alpha \in \Gamma}\left(G, T_{\alpha}\right)$. The embedding of $G$ into $\mathscr{G}$ along the diagonal is an algebraic embedding. The relative topology on $G$ in $\mathscr{G}$ is the supremum topology on $G$ relative

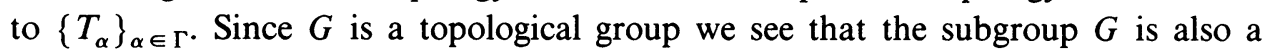
topological group when given this topology. Hence $\mathscr{L}$ is closed under the operation $T_{\alpha} \vee T_{\beta}$ where $T_{\alpha} \vee T_{\beta}$ denotes the supremum topology $T_{\alpha}$ and $T_{\beta}$.

Now suppose $T_{\alpha}$ and $T_{\beta}$ are in $\mathscr{L}$ and $\mathscr{B} \subset \mathscr{L}$ is the collection of all topologies $T_{\gamma}$ in $\mathscr{L}$ that satisfy $T_{\gamma} \subseteq T_{\alpha}$ and $T_{\gamma} \subseteq T_{\beta} . \mathscr{B} \neq \varnothing$ since the indiscrete topology on $G$ is in $\mathscr{L}$. We define $T_{\alpha} \wedge T_{\beta}=\left(\vee_{\gamma \in \mathscr{B}} T_{\gamma}\right)$. Certainly $\mathscr{L}$ is closed under $\wedge$ and hence $(\mathscr{L}, \vee, \wedge)$ forms a complete lattice. It should be noted that this lattice is different from the usual lattice of topologies on a set $X$ since the intersection of two group topologies may not be a group topology.

Let $H$ be a topological group contained in a group $G$. A topology which makes $G$ a topological group inducing the given topology on $H$ is called an extending topology. The set of all extending topologies from $H$ to $G, \mathscr{E}$, is a complete subsemilattice of the complete lattice of group topologies on $G$. The purpose of this paper is to further study $\mathscr{E}$.

Definition. A group topology for $H$ is said to be translatable if and only if for every neighborhood $U$ of $e$ in $H$ and every $g \in G$, the set $g U g^{-1}$ contains a neighborhood of $e$ in $H$. If $t$ is a translatable topology on $H$ we define the translation topology $T_{H}^{*}$ to be that topology on $G$ which has $\{g U \mid g \in U$ and $U \in t\}$ as a basis.

One way to create a topology on $G$ is to find a homomorphism from $G$ to a topological group $G^{\prime}$. The weak topology on $G$ relative to this homomorphism will make $G$ into a topological group also. Occasionally this topology will be an extending topology. Let $H$ be a subgroup of $G$ endowed with the translatable

Received by the editors May 16, 1985 and, in revised form, June 18, 1985.

1980 Mathematics Subject Classification. Primary 22A99.

Key words and phrases. Lattice, group topologies.

C1986 American Mathematical Society $0002-9939 / 86 \$ 1.00+\$ .25$ per page 
topology $t$. Let $f$ be a homomorphism from $G$ onto the topological group $G^{\prime}$ and let $H^{\prime}=f(H)$. Let $E$ be the closure of $\{e\}$ in $H$.

THEOREM 1. The weak topology on $G$ induced by $f$ is an extending topology for $H$ if and only if $f$ is continuous and open (as a map from $H$ to $H^{\prime}$ ) and $H \cap \operatorname{ker} f \subseteq E$.

Proof. Let $T$ be the weak topology on $G$ induced by $f$ and suppose that $T \in \mathscr{E}$. If $x \in H \cap \operatorname{ker} f$ and $U$ is an open neighborhood of $x$ in $H$, then there exists an open set $U^{\prime}$ in $H^{\prime}$ such that $U=H \cap f^{-1}\left(U^{\prime}\right)$. But $e^{\prime} \in U^{\prime}$ and hence $e \in U$.

Now suppose that $H \cap \operatorname{ker} f \subseteq E$ and $f$ is continuous and open as a map from $H$ to $H^{\prime}$. Let $U \in t$. Then $U^{\prime}=f(U)$ is open in $H^{\prime}$. Let $x \in H \cap f^{-1}\left(U^{\prime}\right)$. We can find a $y \in U$ such that $f(x)=f(y)$ and hence $x y^{-1} \in \operatorname{ker} f$. So if $x y^{-1} \in V$ and $V \in t$, then $e \in V$. But this means that $x \in U$ and hence $U=H \cap f^{-1}\left(U^{\prime}\right)$.

Let $N=\operatorname{ker} f$. Obviously, $N$ is a normal subgroup with $N \cap H=\{e\}$. Such subgroup structure occurs frequently (e.g., if $G$ has a presentation of the form $\left\{a, b \mid a^{n}=b^{m}\right\}$, then $H=[G, G]$ and $N=Z(G)$ satisfy this condition). Since we are trying to create new extending topologies on $G$, we may hope that any extending topology on $G$ will induce a topology on $G / N$ that can be used to create a weak topology on $G$ that also extends the topology on $H$. In general this will not work since we cannot be sure that $\left.f\right|_{H}$ is a homeomorphism where $f: G \rightarrow G / N$ is the natural map.

As an example of this, suppose that $G=\mathbf{R}^{1}$ and $N=\mathbf{Q}$. If $H$ is a linear complement to $\mathbf{Q}$ when $\mathbf{R}^{\mathbf{l}}$ is regarded as a vector space over $\mathbf{Q}$, then the usual topology on $H$ will be translatable since $\mathbf{R}^{1}$ is abelian. But the weak topology on $\mathbf{R}^{1}$ relative to the natural map $f: \mathbf{R}^{1} \rightarrow \mathbf{R}^{1} / Q$ is the indiscrete topology.

Suppose that $H$ is any subgroup and that $N$ is a normal subgroup with the property that $H \cap N=\{e\}$. Certainly the multiplication map $H \times N \rightarrow H N$ is bijective. If $H$ and $N$ are assigned group topologies, then this map induces a topology on $H N$, called the product topology, which may or may not be a group topology for $H N$. We note that a group topology for $H N$ is a product topology if and only if the map of $H$ to $H N / N$ is a homeomorphism.

THEOREM 2. Suppose $H$ and $N$ are endowed with topologies such that the product topology for $H N$ is a group topology. Then this topology for $H N$ is translatable if the given topologies for both $H$ and $N$ are translatable. On the other hand, if the topology for $H N$ is translatable, then the topology for $N$ is translatable.

We observe that the indiscrete topology on a normal subgroup is always translatable. If $H$ is normal, then the product topology for $H N$ will be a group topology. So if $t$ is a translatable topology for $H$ and $N \cap H=\{e\}$, then there is an extending topology which has the indiscrete topology as the relative topology on $N$.

The collection of normal subgroups $\mathscr{N}$ with the property that if $n \in \mathscr{N}$ then $n \cap H=\{e\}$ is a nonempty collection. Thus if we partially order $\mathscr{N}$ by set inclusion, we can use Zorn's Lemma to find maximal normal subgroups $N$ with $N \cap H=\{e\}$. As we shall soon see, these maximal subgroups will give us the best insight to the structure of $\mathscr{E}$. 
Let $H$ be normal. Then $T \in \mathscr{E}$ where $T$ is generated using $\{g U \mid U \in t \times\{N, \varnothing\}$, $g \in G\}$ as a basis. Let $\tau_{N}$ be the topology on $G / H$ which is generated by using the natural map from $(G, T)$ to $\left(G / H, \tau_{N}\right)$. The set of topologies on $G / H$ which make $G / H$ into a topological group and which lie between $\tau_{N}$ and the discrete topology on $G / H$ forms a lattice $L_{N}$ contained in the lattice of all continuous topologies that exist on $G / H$. If $n$ is a normal subgroup of $G$ with $n \subset N$, then a moment's reflection will show that $L_{n} \subset L_{N}$ where $L_{n}$ is defined in the same fashion as $L_{N}$.

THEOREM 3. If $N \subset G$ is a normal subgroup with $N \cap H=\{e\}$, then the semilattice $\mathscr{E}$ contains a sublattice isomorphic to $L_{N}$.

Proof. As we have seen, $T \in \mathscr{E}$ and the natural map from $(G, T)$ to $\left(G / H, \tau_{N}\right)$ puts a continuous topology on $G / H$. By [1] we know that for every topology $\tau$ in $L_{N}$ there is a unique topology in $\mathscr{E}$ that induces $\tau$ and is finer than $T$. Let $\mathscr{L}_{N}$ be this collection of topologies. Since $\mathscr{L}_{N}$ includes both $T$ and the translation topology and since we have an order-preserving bijection between $\mathscr{L}_{N}$ and $L_{N}$, it is clear that $\mathscr{L}_{N}$ is isomorphic to $L_{N}$.

As an example of this theorem's applications, let $G$ be the torus knot group with the presentation $\left\{a, b \mid a^{n}=b^{m}\right\}$ with $(n, m)=1$. We place the residually finite topology on $G$ as described in [2]. If $H=[G, G]$, we then have a relative topology defined on $H$ which we call $t$. Let $N$ be the center of $G$. Of course, any group topology placed on the center of a group will be a translatable topology. In this example $N$ is isomorphic to $\mathbf{Z}$ and clearly $N \cap H=\{e\} . G / H \cong \mathbf{Z}$ is the homology group associated with the torus knot space, and the image of $N$ in $G / H$ is the subgroup $\{n m x \mid x \in \mathbf{Z}\}$. The indiscrete topology on $N$ and hence the indiscrete topology on the subgroup $\{n m x \mid x \in \mathbf{Z}\}$ translates to make $G$ and $G / H$ into topological groups. Let $\left\{p_{1}, p_{2}, \ldots, p_{j}\right\}$ be the collection of primes that divide the composite number $\mathrm{nm}$, and let $\mathscr{P}$ be any collection of primes that contain $\left\{p_{1}, p_{2}, \ldots, p_{j}\right\}$ as a subset. There is a unique topology $\tau_{\mathscr{P}}$ on $\mathbf{Z}$ which is the supremum topology of $\{p$-adic topology $\mid p \in \mathscr{P}\}$. If $\mathscr{P} \neq \mathscr{P}^{\prime}$, then $\tau_{\mathscr{P}} \neq \tau_{\mathscr{P}}$. Therefore $\mathscr{E}$ contains uncountably many topologies corresponding to the $\tau_{\mathscr{P}}$ topologies on Z. Of course, there are coarser topologies on $G / H$ which fail to be Hausdorff, but which also have their corresponding topology in $\mathscr{E}$. As pointed out in [3], there are topologies on $G / H$ which are finer than the subgroup topology, but which are not the discrete topology. They also have their counterparts in $\mathscr{E}$. Although we have discovered many topologies in $\mathscr{L}_{N} \subset \mathscr{E}$ and their relationship to each other, we have not found the ultimate structure of $\mathscr{E}$. In this example we note that the residually finite topology on $G$ is not an element of $\mathscr{L}_{N}$.

If $T \in \mathscr{L}_{N} \subset \mathscr{E}$ for some normal subgroup $N \subset G$, we shall call $T$ a normal extension topology. One might hope that every topology in $\mathscr{E}$ is a normal extension topology. This is unfortunately not the case. Let $G=\mathbf{R}^{1}$ and $H=\mathbf{Z} \subset \mathbf{R}^{1}$. We place the discrete topology $t$ on $\mathbf{Z}$ and consider the semilattice $\mathscr{E}$.

Let $N$ be a linear complement to $\mathbf{Q}$ when $\mathbf{R}^{1}$ is regarded as a vector space over $\mathbf{Q}$. Suppose that $n / m \in N \cap \mathbf{Q}$. Certainly $n \in N \cap \mathbf{Z}$ and hence $N \cap \mathbf{Q}=\{0\}$. The coarsest normal extension topology we can obtain on $\mathbf{R}^{\mathbf{l}}$ using $N$ is the topology 
obtained by placing the indiscrete topology on $N$, the discrete topology on $\mathbf{Z}$, the resulting product topology on $N \times \mathbf{Z}$ and translating this topology throughout $\mathbf{R}^{1}$. But if we place the indiscrete topology on $N$, the usual topology on $\mathbf{Q}$, the resulting product topology on $N \times \mathbf{Q}$ and translate this topology throughout $\mathbf{R}^{\mathbf{1}}$, we will obtain a coarser topology.

The authors wish to thank Douglass Grant and the referee for helpful comments with respect to this paper.

\section{REFERENCES}

1. B. Clark and V. Schneider, The extending topologies, Internat. J. Math. Math. Sci. 7 (1984), 621-623.

2. __ All knot groups are metric, Math. Z. 187 (1984), 269-271.

3. E. Hewitt and K. Ross, Abstract harmonic analysis. I, Springer-Verlag, New York, 1963.

Department of Mathematics, University of SOUTHWestern Louisiana, LafayetTe, Louisiana 70504 